\title{
Structure and Properties of Multiwall Carbon Nanotubes/Polystyrene Composites Prepared via Coagulation Precipitation Technique
}

\author{
I. N. Mazov, ${ }^{1,2}$ V. L. Kuznetsov, ${ }^{1,2}$ D. V. Krasnikov, ${ }^{2}$ N. A. Rudina, ${ }^{1}$ A. I. Romanenko, ${ }^{2,3}$ \\ O. B. Anikeeva, ${ }^{2,3}$ V. I. Suslyaev, ${ }^{4}$ E. Yu. Korovin, ${ }^{4}$ and V. A. Zhuravlev ${ }^{4}$ \\ ${ }^{1}$ Department of Physical Methods of Investigations, Boreskov Institute of Catalysis, Novosibirsk, Russia \\ ${ }^{2}$ Physical Faculty, Novosibirsk State University, Novosibirsk 630090, Russia \\ ${ }^{3}$ Department of Thermodynamic Investigations, Nikolaev Institute of Inorganic Chemistry, Novosibirsk 630090, Russia \\ ${ }^{4}$ Faculty of Radiophysics, National Research Tomsk State University, Tomsk 634050, Russia
}

Correspondence should be addressed to I. N. Mazov, ilya.mazov@gmail.com

Received 9 February 2011; Revised 25 April 2011; Accepted 28 April 2011

Academic Editor: Baoquan Sun

Copyright ( 2011 I. N. Mazov et al. This is an open access article distributed under the Creative Commons Attribution License, which permits unrestricted use, distribution, and reproduction in any medium, provided the original work is properly cited.

\begin{abstract}
Coagulation technique was applied for preparation of multiwall carbon nanotube- (MWNT-)containing polystyrene (PSt) composite materials with different MWNT loading (0.5-10 wt.\%). Scanning and transmission electron microscopies were used for investigation of the morphology and structure of produced composites. It was shown that synthesis of MWNT/PSt composites using coagulation technique allows one to obtain high dispersion degree of MWNT in the polymer matrix. According to microscopy data, composite powder consists of the polystyrene matrix forming spherical particles with diameter ca. 100$200 \mathrm{~nm}$, and the surface of MWNT is strongly wetted by the polymer forming thin layer with 5-10 nm thickness. Electrical conductivity of MWNT/PSt composites was investigated using a four-probe technique. Observed electrical percolation threshold of composite materials is near to $10 \mathrm{wt} . \%$, mainly due to the insulating polymer layer deposited on the surface of nanotubes. Electromagnetic response of prepared materials was investigated in broadband region (0.01-4 and 26-36 GHz). It was found that MWNT/PSt composites are almost radiotransparent for low frequency region and possess high absorbance of EM radiation at higher frequencies.
\end{abstract}

\section{Introduction}

Carbon nanotubes attract high attention as perspective material due to their high mechanical strength, Young's modulus, thermal and electrical conductivity, and chemical stability. One of the most interesting ways of application of multiwall carbon nanotubes (MWNT) is their usage for synthesis of MWNT-reinforced composite materials. Introduction of carbon nanotubes in polymer matrix leads to significant increase of material's properties, such as mechanical strength, electrical conductivity, fracture toughness, and electromagnetic shielding properties [1-3]. In recent decade many works were done in the field of the development of preparation techniques and investigation of properties of MWNT-reinforced plastic composites, based on such matrices as epoxy resin [4], polymethylmethacrylate [5], polyurethanes [6], polystyrene [7], and polypropylene and polyethylene [7, 8] Nowadays several preparation methods were successfully used for synthesis of CNT-containing composites, melt mixing [9], solvent cast technique [10], in situ polymerization $[11,12]$, coagulation precipitation [13], and so forth. Among these methods coagulation precipitation technique described by $\mathrm{Du}$ et al. [14] is one of the most promising because of its experimental simplicity, potential scalability, and ability for usage for synthesis of various thermoplastic-based polymer composites. In this method the filler material and polymer matrix precursor are dissolved in appropriate medium, treated (with ultrasound, mechanical stirring, or any other necessary technique) for disaggregation and distribution of filler, and produced mixture is poured in 
appropriate solvent, providing dissolution of the suspension medium with further precipitation of the matrix material with incorporated filler. Using this technique, it is possible to achieve high degree of distribution of filler (e.g., carbon nanotubes) in the matrix due to relatively high amount of the solvent with low viscosity as compared with pure matrix. Successful application of the coagulation precipitation technique depends strongly on the right choice of the solvent medium and disaggregation method.

In the present work coagulation precipitation technique was applied for preparation of MWNT-containing polystyrene composites with various CNT content, and investigation of their morphological, structural, electrophysical, and electromagnetic properties was performed.

\section{Experimental and Methodology}

Multiwall carbon nanotubes (MWNT) with average diameter 20-22 nm were produced on site by CVD decomposition of ethylene over bimetallic FeCo catalysts at $680^{\circ} \mathrm{C}$. Synthesized MWNTs were purified by reflux with $\mathrm{HCl}(1: 1)$, rinsed with distilled water, and dried in air at $60^{\circ} \mathrm{C}$ overnight.

Polystyrene (PSt) with molecular weight ca. 75000 100000 was dissolved in dimethylformamide (DMF) to form concentration $0.05 \mathrm{~g} / \mathrm{mL}$.

Coagulation technique described elsewhere [15] for preparation of MWNT/PMMA composites was applied for synthesis of MWNT/PSt composites as follows. Necessary amount of purified MWNTs was placed in the watercooled glass reaction vessel, $80 \mathrm{~mL}$ of PSt/DMF solution and $40 \mathrm{~mL}$ of pure DMF were added to MWNT, and the mixture was sonicated $(22 \mathrm{KHz}, 800 \mathrm{~W})$ during 1 hour. After sonication MWNT/PSt/DMF suspension became uniformly black and was poured into ca. $1000 \mathrm{~mL}$ of distilled water (55$60^{\circ} \mathrm{C}$ ). Immediately after mixing with water precipitation of MWNT/PSt composite takes place leading to formation of spongy deposit with color changing from light gray to black depending on MWNT loading. MWNT/PSt composites with MWNT con tent $0.5,1.0,2.0,4.0$, and $10.0 \mathrm{wt} . \%$ were prepared using described technique.

Produced deposit was filtered, rinsed with water $(500 \mathrm{~mL}$ for 3 times) and dried in air at $60^{\circ} \mathrm{C}$ overnight, milled in rotary mill, and dried once more at $60^{\circ} \mathrm{C}$ in vacuum ( $10^{-2}$ torr) for 4 hours until the constant weight. Composite powder was hot pressed at $115-120^{\circ} \mathrm{C}$ between polished steel plates with pressure up to $200 \mathrm{~kg} / \mathrm{cm}^{2}$ to form films with dimensions ca. $60 \mathrm{~mm} \varnothing \times 0.3 \mathrm{~mm}$.

Scanning and transmission electron microscopy investigations were performed using JSM6460LV and JEM-2010 microscopes, correspondingly. For SEM analysis polymer films were broken and glued to the copper plate using silver glue with fresh break upwards. In order to avoid charging effects composites were gold coated with layer thickness 60-80 A. TEM analysis was performed for powder samples deposited on the copper grid from ethanol suspension using horn ultrasonicator.

Electrical conductivity of composite films was measured using four-probe technique in temperature range $4.2-300 \mathrm{~K}$ with silver wire $(0.1 \mathrm{~mm}$ in diameter $)$ contacts attached to the sample using silver glue. The low conductivity threshold of the experimental setup was $10^{-11} \mathrm{~S} / \mathrm{cm}$.

Electromagnetic response properties (reflection, absorbance, and transmission coefficients on power) were measured in $0.01-4$ and $26-36 \mathrm{GHz}$ frequency ranges. For low-frequency range coaxial cell with $16 \mathrm{~mm} \mathrm{O.D.} \mathrm{and}$ $6.95 \mathrm{~mm}$ I.D. connected to the transmittance and reflectance measurement unit R2-4M (Mikran, Russia) was used. High frequency measurement was performed using open horn measurement cell, connected with HP Agilent PNA E8363B vector network analyzer.

\section{Results and Discussion}

Structure of carbon nanotubes was investigated by means of transmission electron microscopy. The typical TEM image of MWNTs used for synthesis of composites is shown on Figure 1.

The average diameter of MWNTs was determined statistically after analysis of 400-450 individual nanotubes on 1015 TEM images and Gatan software package and made the value of 22-24 nm. No additional purification was used for removal of possible excessive amorphous carbon-according to our previous studies [16] the amount of amorphous species on the surface of MWNTs does not exceed 0.5 at.\%. The residual metal particles are situated mainly inside of carbon nanotubes, the quantity of the metal was estimated by $\mathrm{X}$-ray fluorescent analysis and was found to be ca. $0.5 \mathrm{wt} . \%$.

Investigation of the internal structure of composite films was performed using scanning electron microscopy in order to obtain data on the dispersion state of MWNTs in the polystyrene matrix. Optical microscopy was used. On Figure 2 SEM images of fresh breaks of the composite films with MWNT concentration 0.5, 1.0, 2.0, 4.0, and $10 \mathrm{wt} . \%$, and reference pure polystyrene film are shown. Carbon nanotubes are well distributed in the polymer matrix for all concentrations, noting that the increase of CNT content does not lead to formation of visible agglomerates in the bulk volume of the polymer. MWNTs are covered with the polymer layer, which remains intact after breakage of the composite film (see Figure 2(d) e.g.) indicating good wetting of the CNT surface with the polystyrene.

Transmission electron microscopy images of initial MWNT/PSt composite powders are presented on Figure 3. Polystyrene forms spherical particles with approximate diameter $50-200 \mathrm{~nm}$ either separated or linked with each other (Figure 3(a)), which may be freestanding or situated on the surface of nanotubes (Figures 3(b) and 3(c)). The surface of MWNTs is covered with the layer of amorphous polystyrene with thickness up to $10-20 \mathrm{~nm}$ or forming droplike particles on the surface of nanotubes (see Figures 3(b), $3(\mathrm{c})$, and $3(\mathrm{~d}))$. The average value of the contact angle between polystyrene particles and carbon nanotubes lays in range 18-28 degrees, indicating good wetting of the CNT surface with the polymer.

Wetting of the nanotube surface with polymer may be of crucial importance to obtain good disaggregation of 


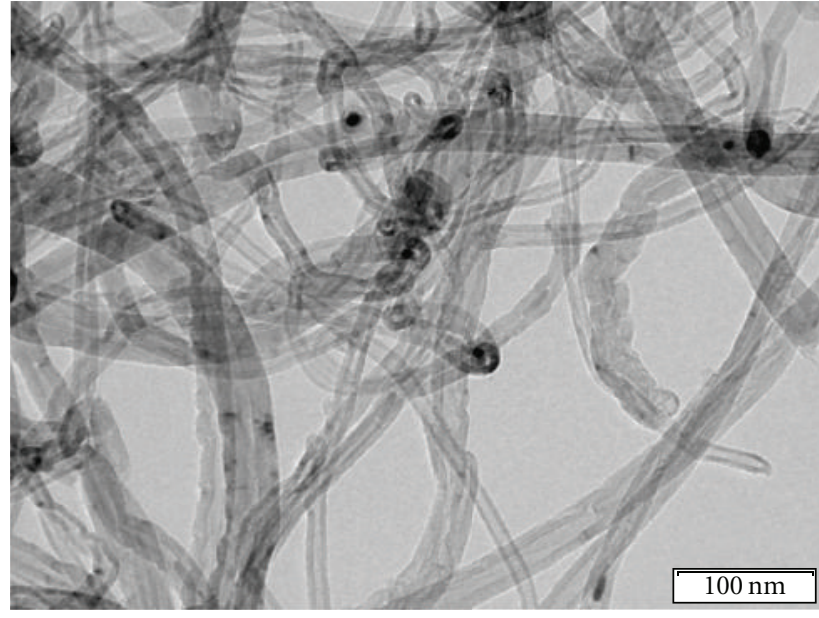

(a)

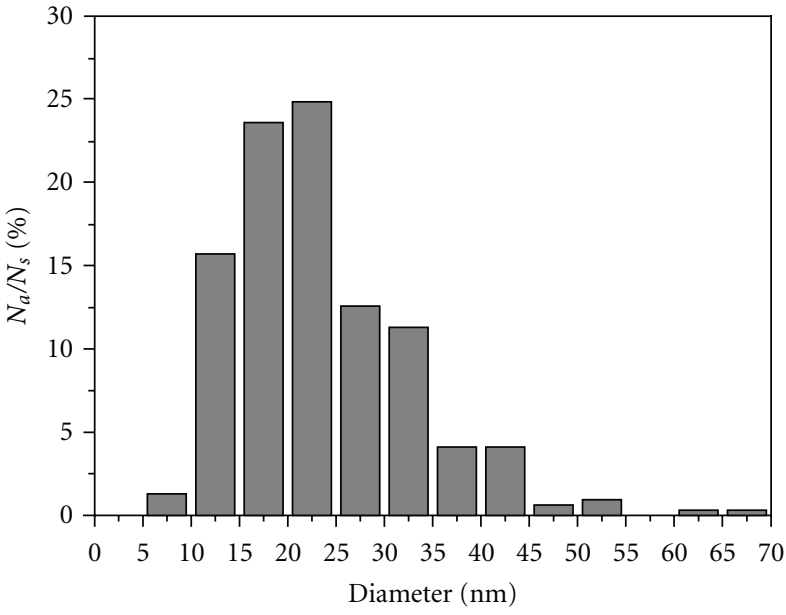

(b)

FIGURE 1: Initial multiwall carbon nanotubes, (a) TEM image of MWNTs, (b) statistical distribution of MWNTs.

MWNTs and subsequent high dispersion state of nanotubes in polystyrene matrix due to change of the interaction behavior from "CNT-polystyrene" in the case of low wetting to "polystyrene-polystyrene" in the case of the high wetting. Nevertheless, the presence of the insulating polymer layer on the surface of conductive nanotube can significantly change the electrical conductivity of resulting composite.

Thus according to the microscopy data it is reasonable to conclude that coagulation precipitation of the polystyrene/MWNT mixture from DMF solution allows one to produce composite material with highly disaggregated nanotubes. As it was early mentioned by $\mathrm{Du}$ et al. [14] for SWNT/PMMA composites precipitation of the dissolved polymer leads to wrapping of the disaggregated nanotubes with polymer chains, thus preventing further agglomeration of CNTs and in our case leading to insulating of the nanotubes by relatively dense polymer layer with thickness comparable with MWNT diameter. Agglomeration of MWNTs during deposition and further film processing does not occur mainly due to the polymer layer formed on the surface of the nanotubes, preventing strong van der Waals interactions between individual CNTs, which are produced from agglomerates during ultrasonication in DMF which is known as a well-dispersing agent for carbon nanostructures [17] because of its high polarity and free electron pair of the nitrogen atom which interacts with distributed $\pi$-system of CNT allowing good wetting of the surface and further disaggregation of tangled agglomerates.

Thus in our case highly dispersed nanotubes are covered with the polymer layer immediately after disaggregation and this may be of crucial importance to further formation of the distributed network of CNTs in the bulk polymer matrix.

Electrical resistivity measurements of MWNT/PSt composite films were performed using four-probe technique, applied earlier for investigation of electrophysical properties of MWNTs and MWNT-containing polymer composites [18]. It is well known that introduction of continuous carbon nanotubes in the dielectric polymer matrix allows to increase conductivity of the resulting composite for several orders of magnitude. Conductive composite materials can be characterized by the percolation threshold which for multiwall carbon nanotubes lays in range from ca. $0.005 \mathrm{wt} . \%$ [19] to 3-4 wt.\% [20] depending on the electrophysical properties of initial nanotubes and peculiarities of the composites' preparation (alignment and dispersion of nanotubes etc.).

Earlier we have observed percolation threshold ca. $0.5 \mathrm{wt} . \%$ for the same type of nanotubes incorporated in the polymethylmethacrylate (PMMA) matrix and prepared using the same coagulation technique [21]. Composite materials investigated in this work show better distribution of MWNTs in the polymer matrix, but at the same time surprisingly low electrical conductivity for these materials was found. Significant conductivity $\left(4.4 \times 10^{-4} \mathrm{~S} / \mathrm{cm}\right)$ was observed only for composite sample with $10.0 \mathrm{wt} . \%$ loading of CNTs; all other samples show electrical resistivity higher than $10^{9} \mathrm{Ohm} / \mathrm{cm}$ which was the sensitivity threshold for the setup used for measurements. For comparison MWNT/PMMA composites with the same type of nanotubes showed higher electrical conductivity

This phenomenon can be described taking into consideration the above-mentioned wetting of the nanotubes' surface and formation of polystyrene layer on it. It is well known that electrical percolation proceeds in systems formed with conductive inclusions in the insulating matrix, but in the case of MWNT/PSt composites the clearly observed 3dimensional network in the bulk volume of the polymer is constructed of nonconductive objects due to the strong wetting occurring between carbon nanotube surface and polystyrene and subsequent covering of the surface of each nanotube with polystyrene layer. In this case electrical conductivity may occur only for relatively dense systems with high CNT content in which numerous potential electric contacts are presented. Despite high disaggregation degree and homogeneous distribution of MWNTs for composites with 


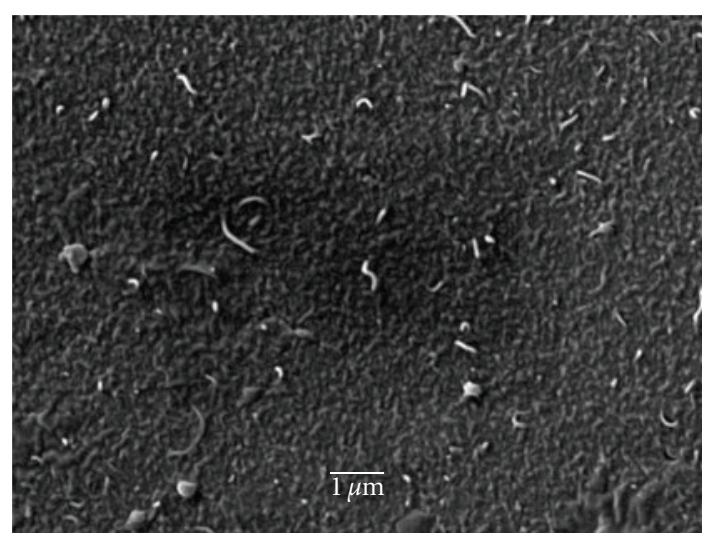

(a)

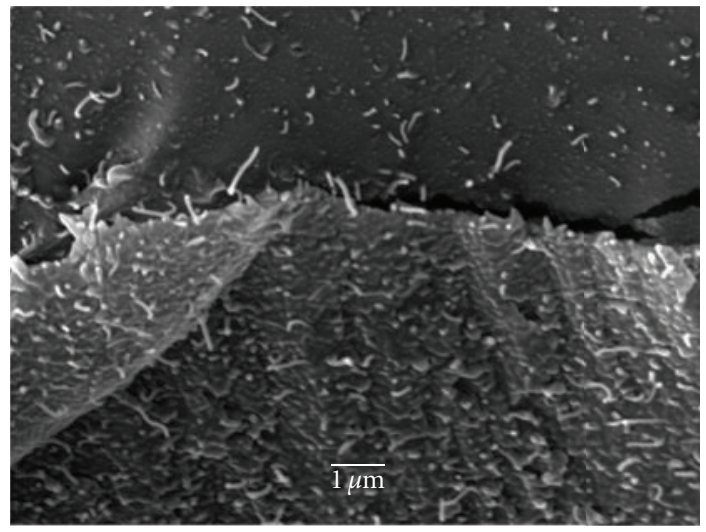

(c)

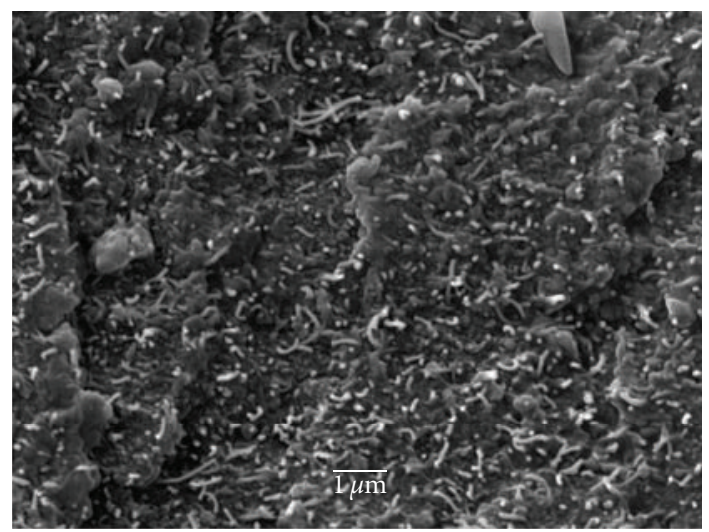

(e)

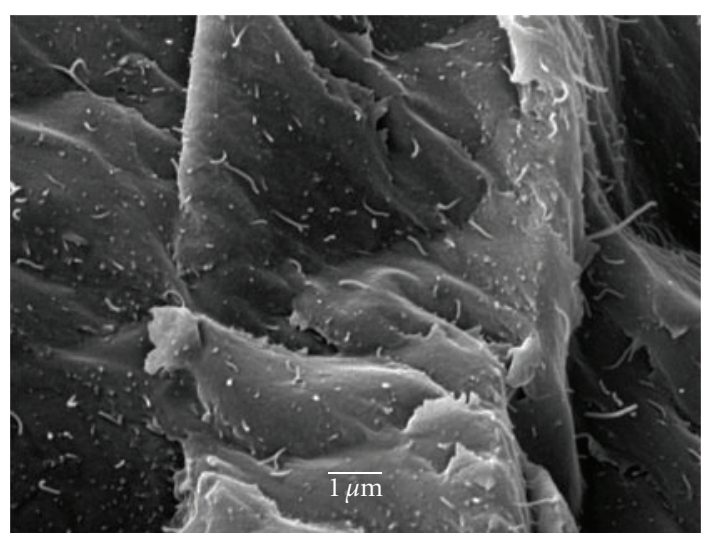

(b)

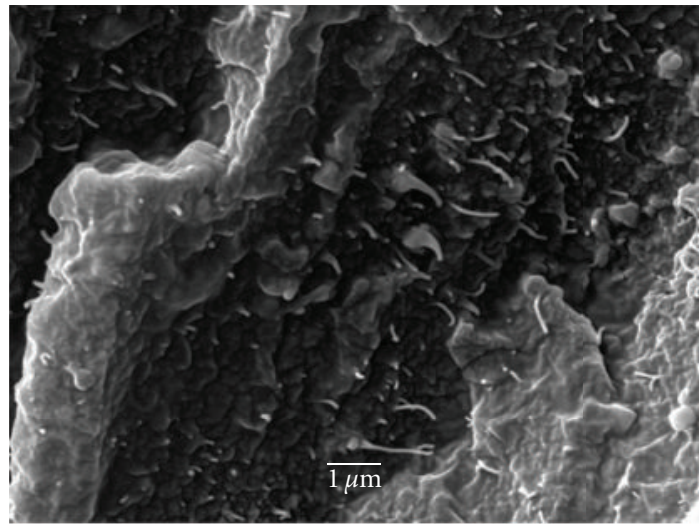

(d)

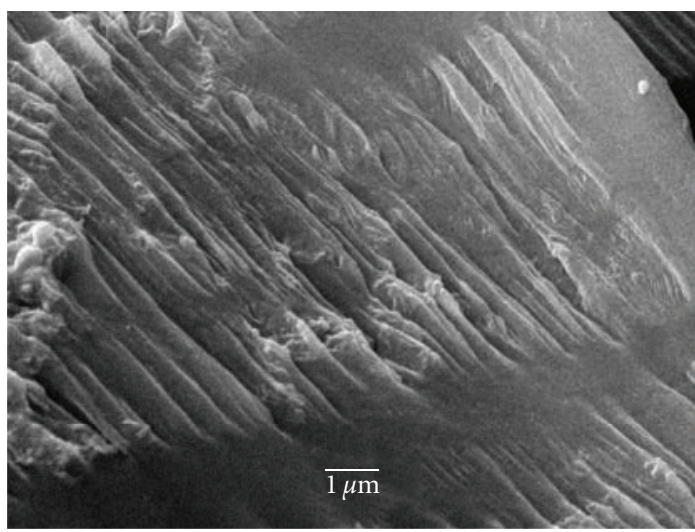

(f)

FIGURE 2: SEM micrographs of the MWNT/PSt composite films (gold-coated fresh breaks) with CNT loading 0.5 (a), 1.0 (b), 2.0 (c), 4.0 (d), and 10.0 (e) wt.\%. On Figure (f) pure PSt film is shown for comparison.

low concentrations of nanotubes, formation of geometrical CNT network is not enough to form electrically conductive system.

Nevertheless, this phenomenon may be used for the design of CNT-based composites with tailorable properties, for example nonconductive coatings with enhanced mechanical, tribological, and thermal properties or for synthesis of EM-shielding coating with low reflection ability due to low electrical conductivity.
Electromagnetic response of produced composites was investigated in frequency range $0.01-4$ and $26-36 \mathrm{GHz}$. In the low frequency region reflection and transmission coefficients for composite materials were measured directly in the coaxial cell, and it was found that nonconductive composites are almost radiotransparent in frequency range $0.01-4 \mathrm{GHz}$. In the microwave frequency region $26-36 \mathrm{GHz}$ transmission $(T)$ and reflection $(R)$ coefficients were measured using open horn measurement sell. Absorption coefficient $(A)$ of the 


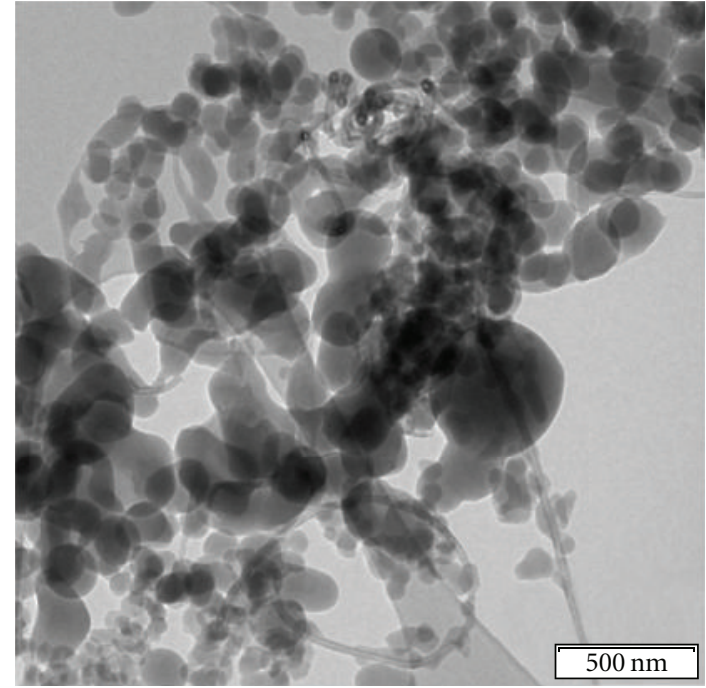

(a)

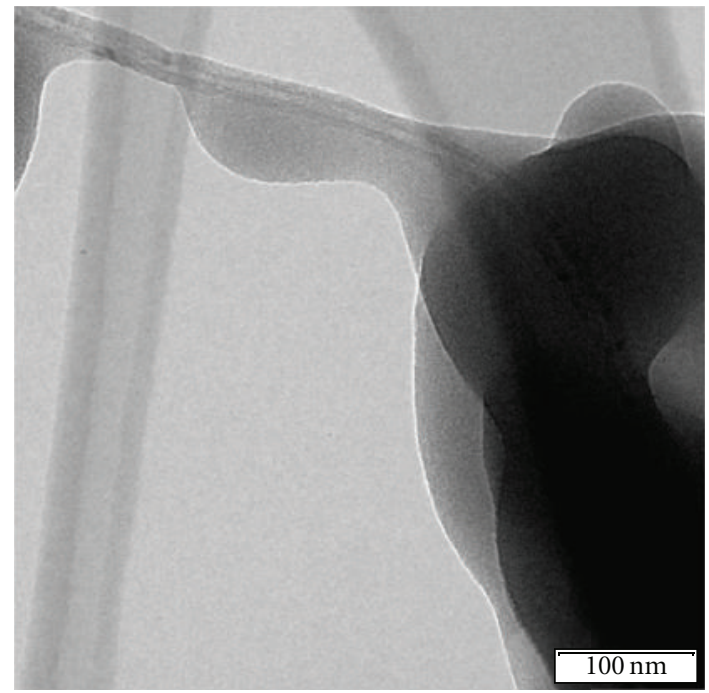

(c)

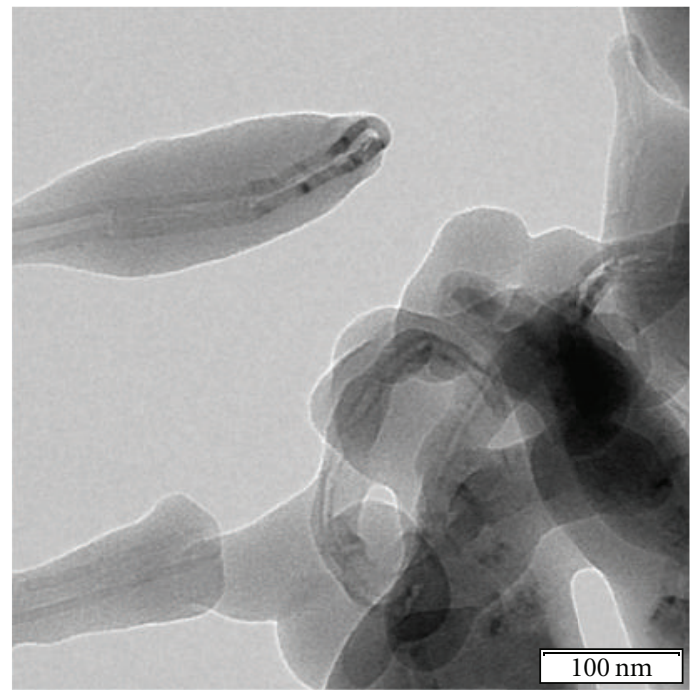

(b)

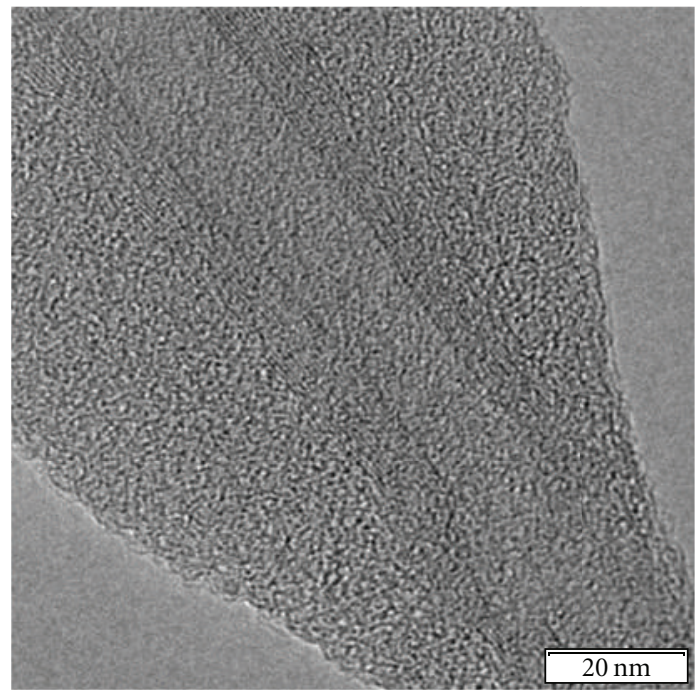

(d)

FIGURE 3: TEM images of MWNT/PSt composite powders with different MWNT loading. Carbon nanotubes covered with polystyrene. (a) 2 wt.\% of MWNTs in PSt, 2-10 wt.\% of MWNTs in PSt, 3, 4-0.5 wt.\% of MWNTs in PSt. The polymer is presented in form of spherical particles (a) and the layer on the surface of CNT (b, c, d).

EM radiation in the sample can be calculated as $A(\%)=$ $100-R-T$.

Data on the dependences electromagnetic response of MWNT/PSt composites with various MWNT loading at fixed frequencies are shown in Figure 4. Increase of the EM radiation frequency leads to the simultaneous growth of the absorbance coefficient and, what is the most surprising, to the sharp diminishing of the reflectance of EMI even for composites with high MWNT loading.

EM properties of all composite materials are changing with increase of the MWNT content in the material. Only slight changes can be seen for the composite with $0.5 \mathrm{wt} . \%$ MWNT loading - it is almost radiotransparent in all frequency range. Increase of the MWNT content leads to drastic increase of the EM absorbance and reflectance with subsequent drop of the EM transmittance coefficient. It is noteworthy that absorbance coefficient $A$ for the samples with high MWNT loading increases more sharply as compared with the reflectance coefficient $R$ especially for higher frequencies.

Analyzing data on the EM response of the composites versus MWNT loading and frequency, one can see percolation-like behavior. At the same time no significant electrical conductivity was observed for all samples excluding $10 \mathrm{wt}$.\% sample. Such behavior can be explained taking into consideration structural data for MWNT/PSt composites: (a) high wetting of MWNT surface with polystyrene allows one to obtain high degree of MWNT distribution in the polymer body with formation of the percolative 3-dimensional nanotube network; (b) the surface of each individual 


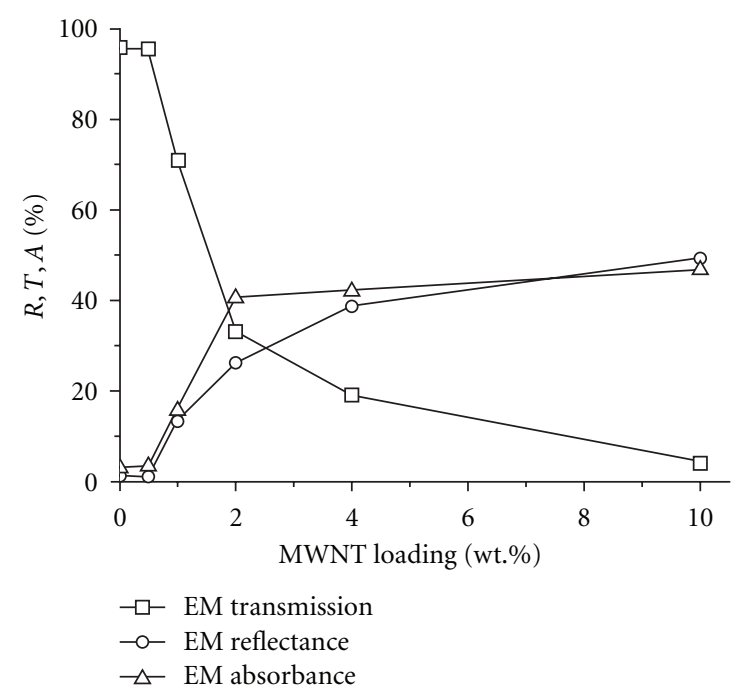

(a)

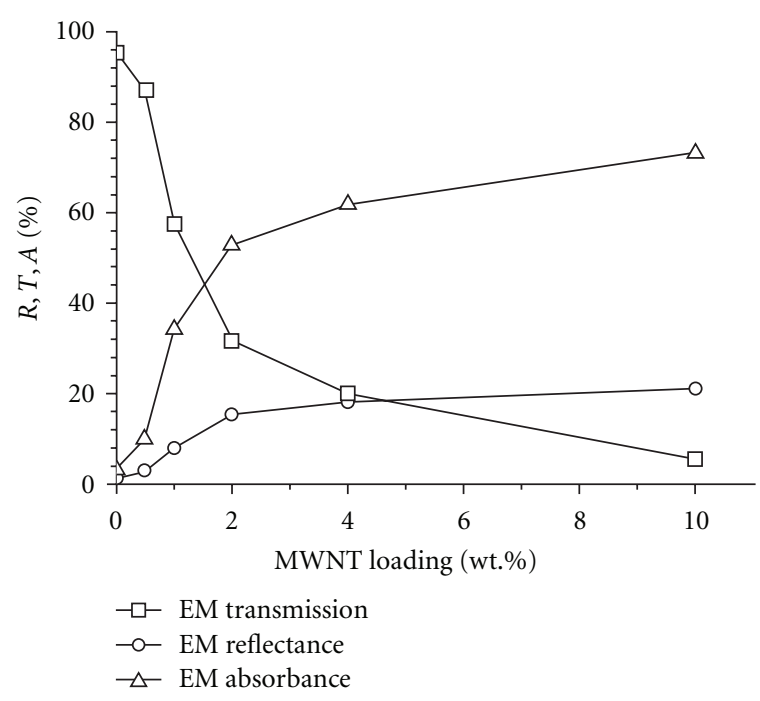

(b)

FIGURE 4: Electromagnetic response of MWNT/PSt composites at fixed frequency versus MWNT loading. (a) EM response at 29 GHz, (b) EM response at $34 \mathrm{GHz}$.

nanotube is covered with the polystyrene thus the abovementioned nanotube network is consisted of insulating objects; (c) the surface conductivity cannot be measured at low MWNT loadings due to insulation between nanotubes on the surface and in the volume of the polymer composite; (d) microwave probing of such material leads to high EM absorbance and relatively low reflectance from the bulk volume of the material.

The last point should be considered separately. The characteristic wavelength for the microwave radiation is $\sim 0.7 \mathrm{~cm}$, which is much higher than the size of the individual nanotube and MWNT agglomerate and the thickness of the composite sample. Thus it is possible to suggest that incident EM wave interacts with the whole composite sample, and this interaction is facilitated due to the absence of surface conductivity and possible skin effects.

Earlier we have observed the effect of high EM shielding for MWNT/PMMA composite materials with the same MWNT loadings in the microwave region [22]. In the case of PMMA-based composites the surface wetting of nanotubes was low, and the surface conductivity of all samples was higher as compared with PSt-based composites with electrical percolation threshold at ca. $0.5 \mathrm{wt} . \%$ of MWNTs.

Thus for conductive MWNT/PMMA samples observed earlier high shielding efficiency was achieved mainly due to the reflectance of EM radiation from the surface of the composite film.

In the case of low-conductive MWNT/PSt composite materials high electromagnetic response may be attributed with various mechanisms of dissipation of EM radiationsuch as mechanical resonances of disaggregated carbon nanotubes in polymer media, metamaterial-like absorbance due to formation of inductance-capacitor (LC) circuits in the carbon nanotube network.
The detailed clarification of the nature of strong EM absorbance by low-conductive MWNT-composites needs further detailed investigations.

\section{Conclusions}

Polystyrene-based composite materials containing multiwall carbon nanotubes were synthesized via coagulation technique. According to electron microscopy study carbon nanotubes are well distributed in the polystyrene matrix forming 3-dimensional uniform network.

High wetting of the nanotube surface with polymer results in formation of the polystyrene layer with thickness $5-15 \mathrm{~nm}$ just after the coagulation, the polymer on MWNTs. Finally that prevents electrical contacts between nanotubes in the composite. The insulation of each nanotube is the main reason of low electrical conductivity for low loadings of CNTs in composite neither shortening or dispersion state of CNT.

Significant electrical conductivity was observed only for materials with $10 \mathrm{wt} . \%$ of MWNT content with corresponding conductivity value $4.4 \times 10^{-4} \mathrm{~S} / \mathrm{cm}$. We have found that EM response of MWNT/PSt composite materials with high CNT loading has high contribution of absorbance in $\mathrm{GHz}$ frequency range in contrast to highly conductive MWNT/PMMA composites investigated earlier where the main contribution into the EM response is reflectance.

Electromagnetic response of the CNT/PSt composite does not depend only on the electrical conductivity of the composites-the interaction of EM radiation with nanotubes occurs at low loadings-namely, 1-2 wt.\%, thus allowing to conclude that formation of the insulated network of CNT interacting with EM radiation proceeds even at low CNT loadings (which is in agreement with SEM data concerning formation of uniform CNT distribution in 
polystyrene matrix). Detailed mechanism of such high EM absorbance is not clear and needs further investigations.

MWNT-containing composites with polystyrene matrix can be used for synthesis of tailorable materials with variable electrical and electromagnetic properties.

\section{Acknowledgments}

This work was partially supported by ITSC Project no. B-1708, Russian state Contracts nos. 16.740.11.0016 and 16.740.11.0146, and RFBR Grant no. 11-03-00351-a.

\section{References}

[1] H. Xu, S. M. Anlage, L. Hu, and G. Gruner, "Microwave shielding of transparent and conducting single-walled carbon nanotube films," Applied Physics Letters, vol. 90, no. 18, Article ID 183119, 2007.

[2] G. M. Odegard, S. J. V. Frankland, and T. S. Gates, "Effect of nanotube functionalization on the elastic properties of polyethylene nanotube composites," AIAA Journal, vol. 43, no. 8, pp. 1828-1835, 2005.

[3] Y. Yang, M. C. Gupta, and K. L. Dudley, "Towards costefficient EMI shielding materials using carbon nanostructurebased nanocomposites," Nanotechnology, vol. 18, no. 34, Article ID 345701, 2007.

[4] Y. Zhou, F. Pervin, L. Lewis, and S. Jeelani, "Fabrication and characterization of carbon/epoxy composites mixed with multi-walled carbon nanotubes," Materials Science and Engineering A, vol. 475, no. 1-2, pp. 157-165, 2008.

[5] S.-M. Yuen, C.-M. Ma, C.-Y. Chuang et al., "Effect of processing method on the shielding effectiveness of electromagnetic interference of MWCNT/PMMA composites," Composites Science and Technology, vol. 68, no. 3-4, pp. 963-968, 2008.

[6] R. Zhang, A. Dowden, H. Deng, M. Baxendale, and T. Peijs, "Conductive network formation in the melt of carbon nanotube/thermoplastic polyurethane composite," Composites Science and Technology, vol. 69, no. 10, pp. 1499-1504, 2009.

[7] D. Xu and Z. Wang, "Role of multi-wall carbon nanotube network in composites to crystallization of isotactic polypropylene matrix," Polymer, vol. 49, no. 1, pp. 330-338, 2008.

[8] S. Kanagaraj, F. R. Varanda, T. V. Zhil'tsova, M. S. A. Oliveira, and J. A. O. Simões, "Mechanical properties of high density polyethylene/carbon nanotube composites," Composites Science and Technology, vol. 67, no. 15-16, pp. 3071-3077, 2007.

[9] C. McClory, T. McNally, M. Baxendale, P. Pötschke, W. Blau, and M. Ruether, "Electrical and rheological percolation of PMMA/MWCNT nanocomposites as a function of CNT geometry and functionality," European Polymer Journal, vol. 46, no. 5, pp. 854-868, 2010.

[10] P. Slobodian, A. Lengálová, P. Sáha, and M. Šlouf, "Poly (methyl methacrylate)/multi-wall carbon nanotubes composites prepared by solvent cast technique: composites electrical percolation threshold," Journal of Reinforced Plastics and Composites, vol. 26, no. 16, pp. 1705-1712, 2007.

[11] E. N. Konyushenko, J. Stejskal, M. Trchová et al., "Multi-wall carbon nanotubes coated with polyaniline," Polymer, vol. 47, no. 16, pp. 5715-5723, 2006.

[12] S. J. Park, M. S. Cho, S. T. Lim, H. J. Choi, and M. S. Jhon, "Electrorheology of multiwalled carbon nanotube/ poly(methyl methacrylate) nanocomposites," Macromolecular Rapid Communications, vol. 26, no. 19, pp. 1563-1566, 2005.

[13] S. R. Tatro, L. M. Clayton, P. A. O. Muisener, A. M. Rao, and J. P. Harmon, "Probing multi-walled nanotube/poly(methyl methacrylate) composites with ionizing radiation," Polymer, vol. 45, no. 6, pp. 1971-1979, 2004.

[14] F. Du, J. E. Fischer, and K. I. Winey, "Coagulation method for preparing single-walled carbon nanotube/poly(methyl methacrylate) composites and their modulus, electrical conductivity, and thermal stability," Journal of Polymer Science Part B, vol. 41, no. 24, pp. 3333-3338, 2003.

[15] I. N. Mazov, V. Kuznetsov, S. Moseenkov et al., "Electromagnetic shielding properties of MWCNT/PMMA composites in Ka-band," Physica Status Solidi (B), vol. 246, no. 11-12, pp. 2662-2666, 2009.

[16] A. I. Romanenko, O. B. Anikeeva, T. I. Buryakov et al., "Electrophysical properties of multiwalled carbon nanotubes with various diameters," Physica Status Solidi (B), vol. 246, no. 11-12, pp. 2641-2644, 2009.

[17] F. Inam, H. Yan, M. J. Reece, and T. Peijs, "Dimethylformamide: an effective dispersant for making ceramic-carbon nanotube composites," Nanotechnology, vol. 19, no. 19, Article ID 195710, 5 pages, 2008.

[18] A. I. Romanenko, O. B. Anikeeva, T. I. Buryakov et al., "Influence of surface layer conditions of multiwall carbon nanotubes on their electrophysical properties," Diamond and Related Materials, vol. 19, pp. 964-967, 2010.

[19] A. Moisala, Q. Li, I. A. Kinloch, and A. H. Windle, "Thermal and electrical conductivity of single- and multi-walled carbon nanotube-epoxy composites," Composites Science and Technology, vol. 66, no. 10, pp. 1285-1288, 2006.

[20] W. K. Park, J. H. Kim, S. S. Lee, J. Kim, G. W. Lee, and M. Park, "Effect of carbon nanotube pre-treatment on dispersion and electrical properties of melt mixed multi-walled carbon nanotubes/poly(methyl methacrylate) composites," Macromolecular Research, vol. 13, no. 3, pp. 206-211, 2005.

[21] J. Macutkevic, D. Seliuta, G. Valusis et al., "Dielectric properties of MWCNT based polymer composites close and below percolation threshold," Physica Status Solidi (C), vol. 6, no. 12, pp. 2814-2816, 2009.

[22] I. N. Mazov, V. Kuznetsov, S. Moseenkov et al., "Electromagnetic shielding properties of MWCNT/PMMA composites in Ka-band," Physica Status Solidi (B), vol. 246, no. 11-12, pp. 2662-2666, 2009. 

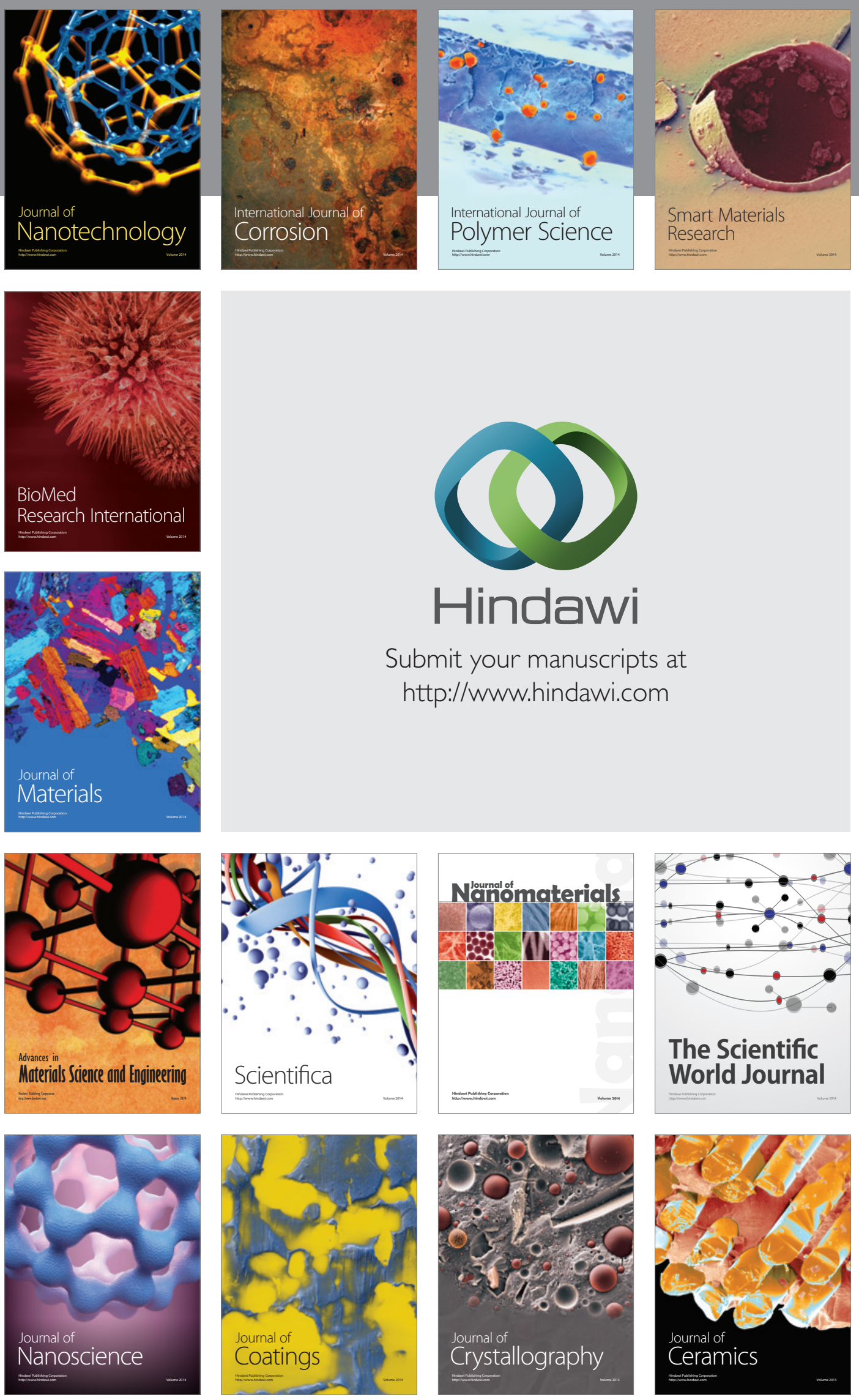

The Scientific World Journal

Submit your manuscripts at

http://www.hindawi.com

\section{World Journal}

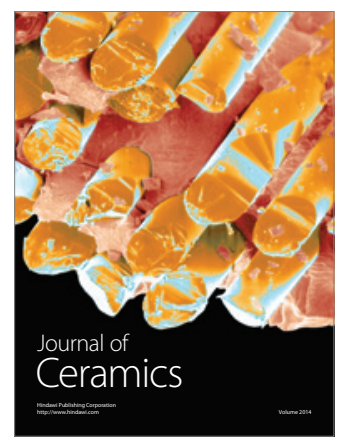

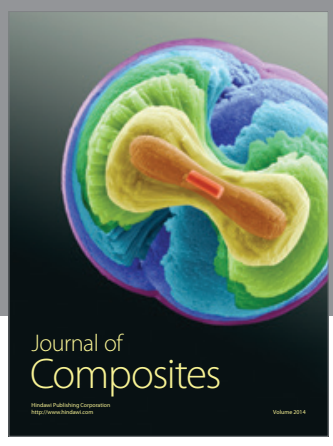
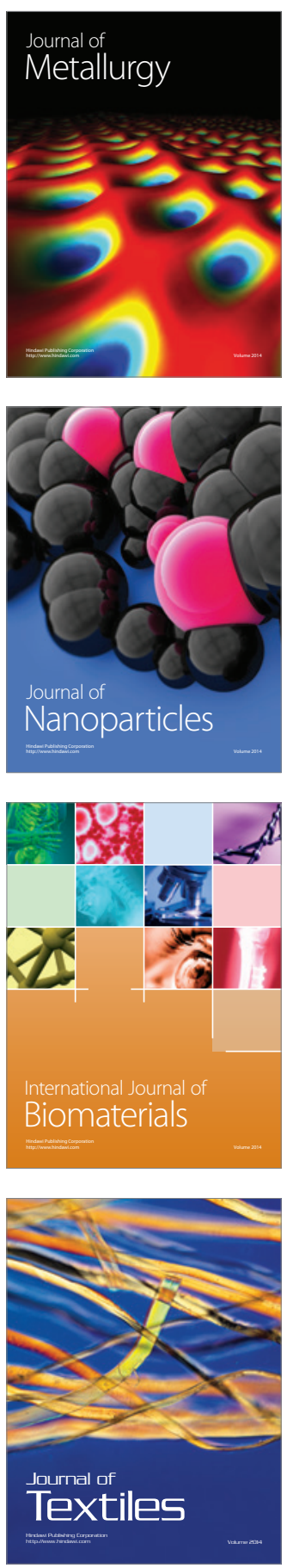